\title{
Frecuencia de complicaciones médicas en un grupo de pacientes mayores de 80 años en hemodiálisis
}

\author{
Pérez-Chávez Paloma Michelle*, Azúa-Díaz Gerardo Gilberto**, Terrones-Saldívar Ma del Carmen**, \\ Rodríguez-Narciso Silvia***
}

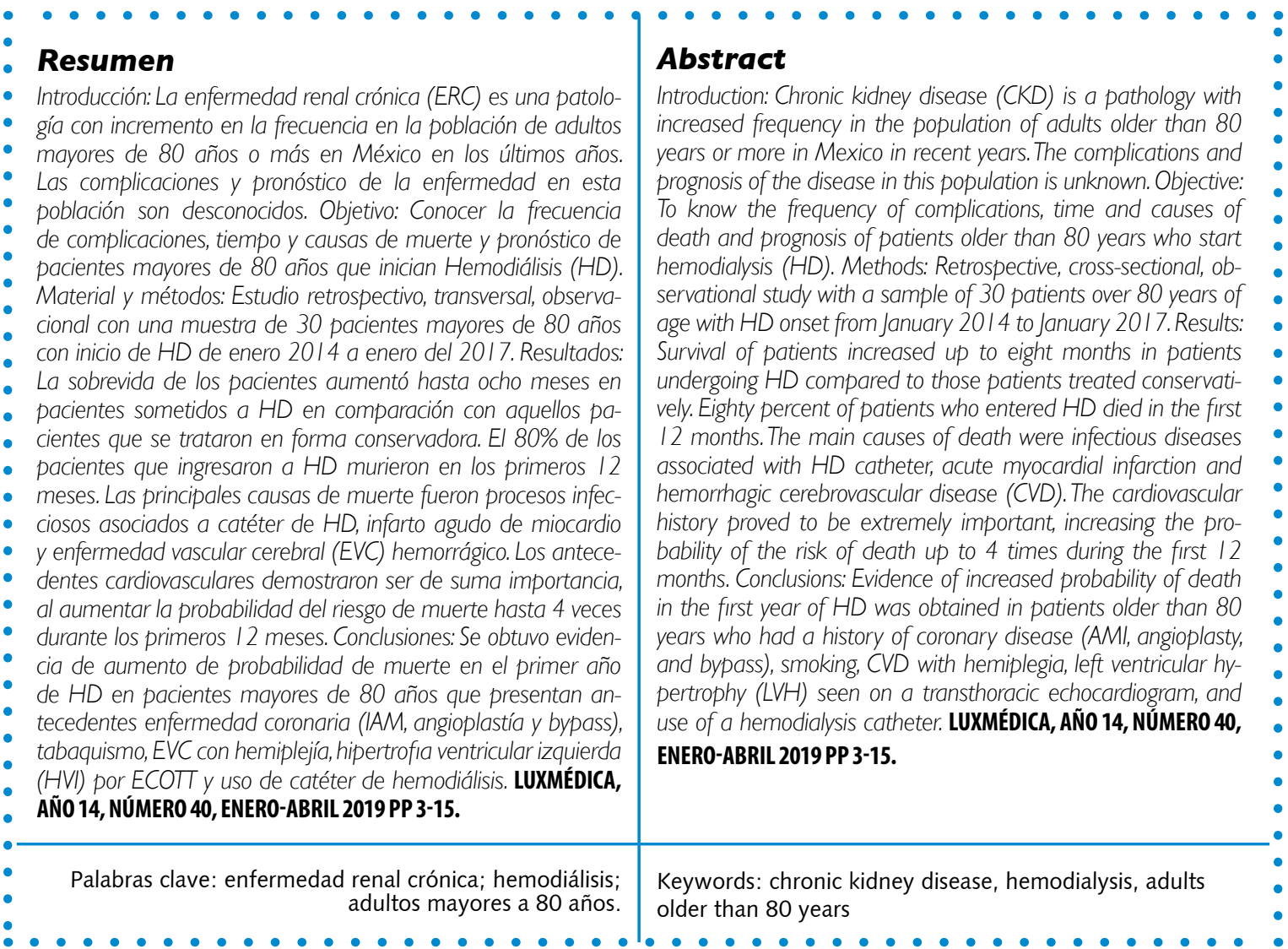

Paloma Michelle Pérez Chávez paloma.pmpc@hotmail.com Estudiante de noveno semestre de la carrera de Medicina del Centro de Ciencias de la Salud de la Universidad Autónoma de Aguascalientes, México.

** Gerardo Gilberto Azúa Díaz geazdi@hotmail.com Profesor del Departamento de Medicina del Centro de Ciencias de la Salud de la Universidad Autónoma de Aguascalientes, México

** Dra. en C. Ma. del Carmen Terrones Saldívar mcterron@correo.uaamx Profesora Investigadora del Centro de Ciencias de la Salud de la Universidad Autónoma de Aguascalientes, México

*** Dra. en C. Silvia Rodríguez Narciso srodrign@correo.uaa.mx Profesor del Departamento de Estadística del Centro de Ciencias Básicas de la Universidad Autónoma de Aguascalientes, México

Fecha de recibido: 10 de abril 2018

Fecha de aceptación: 10 de agosto 2018

Correspondencia: Dr Gerardo Gilberto Azúa Díaz. Departamento de Medicina. Centro de Ciencias de la Salud. Edificio 107 planta alta. Ciudad Universitaria. Universidad Autónoma de Aguascalientes. Avenida Universidad \#940. Código postal 20131. Aguascalientes, Ags., México. Teléfono 449 9108443. Correo electrónico geazdi@hotmail.com 


\section{Introducción}

La enfermedad renal crónica (ERC) es una patología originada por múltiples causas que provoca un alto índice de incapacidad y de morbi-mortalidad. Es la resultante de diversas enfermedades crónico-degenerativas, entre las que se destacan la diabetes mellitus y la hipertensión arterial. En México se desconocen las cifras exactas sobre la población mayor de 80 años afectada por enfermedad renal crónica terminal (ERCT) y que se encuentren en hemodiálisis, a pesar de que es una de las principales causas de atención en hospitalización y en los servicios de urgencias de la mayoría de los hospitales del país. Está considerada como una enfermedad catastrófica debido al número creciente de casos, aunado al incremento de adultos mayores de 80 años, a los altos costos de inversión, recursos de infraestructura y humanos limitados, la detección tardía y altas tasas de morbilidad y mortalidad en programas de sustitución renal. ${ }^{1,2}$

La mayoría de los artículos señalan algunos puntos importantes a considerar para evaluar el tratamiento de pacientes mayores con ERCT; entre éstos se incluyen:

- La esperanza de vida de los pacientes

- El efecto de la ERCT en la esperanza de vida y la calidad de vida

- Comorbilidades presentes en los pacientes ${ }^{2,3}$

Dentro de la información que se conoce, se ha establecido que la esperanza de vida media en EUA, Inglaterra y la mayoría de los países europeos después de los 80 años, es de 7 años para pacientes sin ERCT; en México aún no contamos con información detallada. En lo que se refiere a tipo de terapia sustitutiva en EUA, el tratamiento primario de pacientes mayores (más de 75 años) con ERCT es hemodiálisis (HD) en un $96 \%$.

Los datos del USRDS indican que los pacientes de 65 años o más que se someten a diálisis (ambas terapias) tienen una expectativa de sobrevida de aproximadamente cuatro años, que es marcadamente más baja que la de un paciente de la misma edad sin ERCT. ${ }^{3}$

A pesar de la supervivencia limitada, la mayoría de los pacientes tienen una mejor calidad de vida cuando son sometidos a diálisis, lo que respalda en centros europeos la política de continuar ofreciendo terapia a pacientes mayores con ERCT. La información de 1 año de supervivencia para personas mayores de 80 años con ERCT, es muy variable, oscilando entre el $54 \%$ en Reino Unido y el $90 \%$ en España. De igual manera, la supervivencia media reportada es de 29 meses en Francia y EUA . 4

Con esta información limitada y variable, se debe tener en claro que el uso de terapias de remplazo renal en una población mayor, se debe realizar con el objetivo de mejorar la calidad de vida desde un corto plazo. Lamentablemente en nuestro medio la mayoría 
de la población de 80 años es conducida al uso del tratamiento conservador máximo (TCM) el cual se conoce que establece una supervivencia promedio de 3 a 4 meses, incluyendo medidas de soporte nutricional y médico, reduciendo la fuente de información de este grupo de edad para establecer las comorbilidades asociadas al uso de terapias de reemplazo renal (TRR).

La TRR son las menos estudiadas en este grupo poblacional, al considerarse por muchos clínicos como formas de alargamiento de vida sin beneficio en la calidad de vida de los pacientes, estableciendo falta de información sobre el tema, específicamente en México.

Frecuentemente tanto las comorbilidades del paciente, los antecedentes médicos y las complicaciones derivadas de este tratamiento, así como la infraestructura del sistema de salud actual, son señalados como factores que provocan un alto riesgo de muerte en los pacientes, subestimando el beneficio de una TRR, ya que no se puede establecer una toma de decisiones objetiva acerca del beneficio en calidad de vida, curso, pronóstico y tratamiento de la enfermedad.

Por lo tanto, la diálisis en adultos mayores sigue siendo un desafío y plantea preocupaciones éticas y socioeconómicas. Uno de los motivos de este estudio es obtener información sustentable sobre el grupo de pacientes mayores de 80 años que logre responder de manera objetiva a las interrogantes sobre si es razonable o no ofrecer un tratamiento costoso y complicado a una población que tienen en forma aparente una esperanza de vida limitada. ${ }^{5}$ El objetivo de este trabajo fue conocer la frecuencia de complicaciones, tiempo y causas de muerte en pacientes mayores de 80 años que inician hemodiálisis.

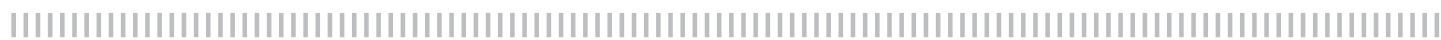

\section{Material y Métodos}

Es un estudio observacional, transversal, retrospectivo. La población en estudio incluyó pacientes que iniciaron TRR, en las unidades de hemodiálisis de Hospital General de Zona Número 2 de la ciudad de Aguascalientes, y la unidad de hemodiálisis del Hospital Regional de Especialidades Número 30 en la ciudad de Mexicali, Baja California, ambos pertenecientes al Instituto Mexicano del Seguro Social. El seguimiento inició en enero 2014 a enero 2017. La mortalidad se registró (en meses) después del inicio de HD.

Se obtiene una muestra no probabilística, por conveniencia de 30 pacientes que cumplieron con los criterios de inclusión.
Se tomaron como criterios de inclusión: 1) Pacientes $\geq 80$ años 2 ) con criterios de inicio de terapia de sustitución renal en hemodiálisis.

Criterios de inicio de terapia de sustitución renal en hemodiálisis:1)TFG $<15 \mathrm{ml} /$ $\mathrm{min} / 1.75 \mathrm{~m}^{2}, 2$ ) Acidosis metabólica (ANION - GAP alto severo, $\mathrm{pH}<7.1$ resistente a tratamiento) 3) Sobrecarga hídrica (ganancia más de $15 \%$ agua corporal total, 4) Hiperkalemia $>65 \mathrm{meq} / \mathrm{l}$ de potasio, 5) Encefalopatía urémica, 6) Pericarditis urémica. Situaciones de urgencia se utilizó un BUN $>100 \mathrm{mg} / \mathrm{l}$ y creatinina sérica $(\mathrm{CrS})>5 \mathrm{mg} / \mathrm{dl} .{ }^{7}$

Se excluyeron a todos los pacientes menores de 80 años con criterios de inicio de terapia en hemodiálisis o diálisis peritoneal 
(DP). No se registran los pacientes en DP ya que durante el desarrollo del estudio no hubo registro de pacientes en el rango de edad con inicio de DP.

Las variables utilizadas fueron obtenidas a partir de los índices ó calculadoras utilizados con mayor frecuencia para establecer riesgos de mortalidad en pacientes con inicio de TRR. Los scores o calculadoras empleadas son: 1 ) Índice de mortalidad de 3 meses en hemodiálisis (o Índice de Estratificación Riesgo), Índice de mortalidad de 6 meses en Hemodiálisis y el Índice de Charlson modificado para sobrevida en TRR. ${ }^{8-10}$

En el estudio se registraron los siguientes datos de los pacientes: 1) datos demográficos (edad, sexo); 2) comorbilidades: diabetes mellitus tipo 2 (DM2) sin daño a órgano blanco/con daño a órgano blanco, tabaquismo, enfermedad coronaria/infarto agudo de miocardio (IAM), insuficiencia cardiaca congestiva (ICC), enfermedad vascular cerebral (EVC) leve/ataque isquémico transitorio (TIA), enfermedad vascular periférica (EVP), demencia, enfermedad pulmonar, enfermedades del tejido conectivo, enfermedad péptica, enfermedad hepática leve/sin hipertensión portal, enfermedad hepática grave/con hipertensión portal, neoplasias (tipo de neoplasia, tumor con metástasis sólidas/tumor sin metástasis sólidas), leucemia, linfoma, SIDA, trastorno cognitivo leve, enfermedad psiquiátrica, disritmia (tipo de disritmia); 3) causa de $E R C T$; 4) referencia por nefrología a TRR o urgencia dialítica; 5) vía para HD: catéter, fístula; 6) albúmina, índice de masa corporal (IMC), fracción de eyección ventricular izquierda (FEVI) obtenida a través de ecocardiograma transtóracico (ECOTT), hipertrofia ventricular izquierda (HVI); 7) clasificación funcional de ICC de New York Heart Association (NYHA), clasificación clínica de Fontaine de enfermedad vascular periférica (EVP); 8) índice de Karnofsky.

La información se obtuvo a través del expediente clínico de la institución (estu- dios de laboratorio y gabinete; valoración funcional de la ICC NYHA; clasificación clínica de Fontaine; Índice de Karnofsky). Se consideró que los pacientes tuvieran comorbilidades si éstas se mencionaban en el expediente clínico o si fueron tratados por esa causa.

Se obtuvieron resultados a través de estadística descriptiva, realizando el análisis de variables cuantitativas con sistema Excell del paquete de software Office 10. La estadística analítica que se utilizó fue a través de la realización de Odds Ratio (OR) definida como la probabilidad de ocurrencia de un evento obtenida mediante el cociente entre la probabilidad de que ocurra el evento y la probabilidad de que no ocurra, a través de la realización de tablas de $2 \times 2$.

Para la descripción del tiempo de muerte a partir del inicio de la hemodiálisis se tomó como punto de corte 12 meses, ya que es el tiempo de supervivencia más bajo demostrado en artículos previos para personas mayores de 80 años en diálisis. Además aproximadamente el $80 \%$ de los pacientes dentro del estudio murieron antes de los 12 meses y $20 \%$ después del año de seguimiento, motivo por el cual se decide utilizar este punto de corte en el tiempo.

Es importante aclarar que una vez establecido el punto de 12 meses y al obtener los datos descriptivos iniciales (complicaciones, causas de muerte, tiempo de muerte), se dispuso a valorar algunos elementos para cada causa de muerte reportada con la intención de obtener una mayor aproximación sobre elementos que pudieron favorecer la muerte, con el motivo de tener un panorama amplio sobre el comportamiento de los pacientes dentro los centros evaluados, reconociendo que no pueden ser extrapolados a otros centros. Se estableció con estadística analítica básica y obtención de OR, la comparación de 1) complicaciones intrahemodiálisis y 2) comorbilidades acompañantes para cada causa de muerte. Además se evaluaron, 
para la principal causa de muerte, los elementos involucrados como el tipo de ac- ceso vascular y microorganismos causantes de procesos infecciosos.

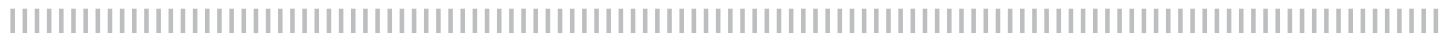

\section{Resultados}

De enero 2014 a enero 2017, se registraron 30 pacientes que iniciaron hemodiálisis con edades de 80 años a 87 años. La edad media al inicio de la hemodiálisis fue de 82.3 años, $40 \%$ mujeres y $60 \%$ hombres. Para el tiempo de muerte a partir del inicio de la hemodiálisis se tomó como punto de corte 12 meses; menor a 12 meses y mayor o igual a 12 meses.

Las principales causas de ERCT fueron: DM2 80\%, Hipertensión Arterial Sistémica (HTA) $13.3 \%$ y Glomerulonefritis Crónica (GNC) $6.6 \%$. Se resalta que $63.3 \%$ de pacientes que murieron antes de los 12 meses la causa de ERC fue DM2 (Tabla 1).

\section{Tabla I}

Causas de ERCT de acuerdo con el tiempo de muerte de la población en estudio

\begin{tabular}{|cccc|}
\hline Tiempo (meses) & \multicolumn{3}{c|}{ Enfermedad } \\
\hline & DM 2 & HTA & GNC \\
\hline <12 meses & $63.3 \%$ & $13.3 \%$ & $3.3 \%$ \\
\hline$>12$ meses & $16.7 \%$ & $0.0 \%$ & $3.3 \%$ \\
\hline
\end{tabular}

Aproximadamente $80 \%$ de los pacien- pués de 12 meses, con un tiempo promedio tes murieron antes de 12 meses y $20 \%$ des- de muerte de $8.2 \pm 4.2$ meses (Tabla 2 ).

\section{Tabla 2}

Tiempo de mortalidad general. Se observa mayor mortalidad en los primeros 12 meses

\begin{tabular}{|lr|}
\hline Tiempo (meses) & Frecuencia (\%) \\
\hline$<12$ meses & $80.0 \%$ \\
\hline$>12$ meses & $20.0 \%$ \\
\hline
\end{tabular}

De los pacientes que murieron antes de los primeros 12 meses, el mayor porcentaje $(25 \%)$ murió entre 7 y 8 meses después del inicio de la hemodiálisis, por diferentes causas (Tabla 3).

\section{Tabla 3}

Porcentajes de mortalidad en el primer año. Durante el primer año se observó mayor mortalidad de 7- 8 meses

\begin{tabular}{|lc|}
\hline Tiempo (meses) & Frecuencia de muerte (\%) \\
\hline$\leq 2$ meses & $8.3 \%$ \\
\hline $3-4$ meses & $20.8 \%$ \\
\hline $5-6$ meses & $20.8 \%$ \\
\hline $7-8$ meses & $25.0 \%$ \\
\hline $9-10$ meses & $12.5 \%$ \\
\hline $11-12$ meses & $12.5 \%$ \\
\hline
\end{tabular}


Las principales causas de muerte de la población en general fueron: 1) sepsis + infección de catéter/sepsis + neumonía,
2) IAM, 3) Enfermedad Vascular Cerebral (EVC) hemorrágico (Tabla 4).

\section{Tabla 4}

\section{Causas de muerte de la población en estudio}

\begin{tabular}{|lc|}
\hline Causa de muerte & Porcentaje (\%) \\
\hline Sepsis + infección de catéter/Sepsis + neumonía & $33.3 \%$ \\
\hline IAM & $30.0 \%$ \\
\hline EVC Hemorrágico & $13.3 \%$ \\
\hline Insuficiencia cardiaca & $6.7 \%$ \\
\hline Accidente & $3.3 \%$ \\
\hline Fractura de cadera & $3.3 \%$ \\
\hline Fractura de columna & $3.3 \%$ \\
\hline Sepsis abdominal & $3.3 \%$ \\
\hline Pancreatitis aguda & $3.3 \%$ \\
\hline
\end{tabular}

La complicación más frecuente causada por hemodiálisis fue la hipotensión intrahemodiálisis con $27 \%$ en los pacientes que murieron antes de los 12 meses. Mostrándose una gran diferencia con los pacientes que murieron después de los 12 meses al no presentarse, y ser la menos frecuente. En orden de frecuencia, la infección de catéter fue la segunda más frecuente con $23 \%$. En los pacientes que murieron después de los 12 meses también fue la más frecuente. Esta complicación se presentó al doble en los pacientes que murieron antes de los 12 meses.

La tercera complicación más frecuente fue hemorragia digestiva alta (HDA)
$17 \%$ en los pacientes que murieron antes de 12 meses, esta misma fue la segunda complicación menos frecuente con $3 \%$ en los pacientes que murieron después de 12 meses.

La cuarta complicación en frecuencia fue la hipertensión intrahemodiálisis, con un $13 \%$ en los pacientes que murieron antes de los 12 meses, y la segunda más frecuente con $7 \%$ en los pacientes que murieron después de 12 meses.

$\mathrm{Al}$ igual que en las causas de muerte, las frecuencia de las complicaciones fue mayor en los pacientes que murieron antes de 12 meses (Tabla 5).

\section{Tabla 5}

\section{Porcentaje de complicaciones por hemodiálisis de la población en estudio}

\begin{tabular}{|ccc|}
\hline Complicación & Tiempo de muerte & (porcentaje \%) \\
\hline & $<\mathbf{1 2}$ meses & $\mathbf{2 1 2}$ meses \\
\hline Infección de catéter & $23 \%$ & $10 \%$ \\
\hline Hipotensión intrahemodiálisis & $27 \%$ & $0 \%$ \\
\hline Hipertensión intrahemodiálisis & $13 \%$ & $7 \%$ \\
\hline Hemorragia digestiva alta & $17 \%$ & $3 \%$ \\
\hline
\end{tabular}




\section{CAUSAS DE MUERTE}

\section{Sepsis + infección de catéter/sepsis + neumonía}

Es la causa más frecuente de muerte de los pacientes (33.3\%); $23.3 \%$ murió antes de 12 meses y $10 \%$ después de 12 meses. La edad promedio de muerte antes de 12 meses fue de 81.9 años y después de 12 meses fue de 82.4 años.

\section{Vía para hemodiálisis}

Al registrar la vía de la HD por catéter y fístula vs. tiempo de muerte, se observó que $20 \%$ de los pacientes en HD con catéter murieron antes de 12 meses y 3.3\% de los pacientes después de 12 meses. La HD realizada con fístula se utilizó en 3.3\% en los que murieron antes de 12 meses, y $6.7 \%$ en pacientes que murieron después de 12 meses. Por lo que el uso del catéter para la HD fue el más frecuente (Tabla 6).

\section{Tabla 6}

Vía para hemodiálisis de acuerdo al tiempo de muerte de la población en estudio

\begin{tabular}{|lcc|}
\hline Vía de hemodiálisis & Muerte $<\mathbf{1 2}$ meses (\%) & Muerte $\mathbf{\geq 1 2}$ meses (\%) \\
\hline Catéter & 20 & 3.3 \\
\hline Fístula & 3.3 & 6.7 \\
\hline
\end{tabular}

\section{Comorbilidades en pacientes con causa de muerte: sepsis + infección de catéter/sepsis + neumonía}

Asimismo, las comorbilidades: DM2, demencia, enfermedad pulmonar y enfermedades de tejido conectivo se encontraban presentes en mayor porcentaje en los pacientes que murieron antes de 12 meses. De los pacientes que murieron por presentar sepsis + infección de catéter/sepsis + neumonía, 10\% presento cáncer: mama, renal y colon, todos con una frecuencia de 3.3\% (Tabla 7).

\section{Tabla 7}

Comorbilidades de pacientes con causa de muerte sepsis + infección de catéter/sepsis + neumonía

\begin{tabular}{|c|c|c|}
\hline & Muerte < 12 meses (\%) & Muerte $\geq 12$ meses (\%) \\
\hline DM2/DM2 con complicaciones & 23.3 & 6.7 \\
\hline Demencia & 10 & 3.3 \\
\hline Enfermedades de tejido conectivo & 6.7 & 6.7 \\
\hline Enfermedades pulmonares & 16.7 & 10 \\
\hline Neoplasias & 6.7 & 3.3 \\
\hline
\end{tabular}

\section{Hemocultivos y cultivos de secreciones}

Los microorganismos más frecuentes encontrados en el hemocultivo, en los que murieron antes de 12 meses, fue Sthapylococcus aureus (S. aureus) $10 \%$; y en los que murieron después de 12 meses fueron S. aureus $3.3 \%$ y Klebsiella pneumoniae (K. pneumoniae) 3.3\%. En los cultivos realizados a partir de muestras de secreción de catéter, el microorganismo más frecuente 
encontrado fue Sthapylococcus epidermidis ( $S$. epidermidis), en los que murieron antes de 12 meses con $10 \%$, y en los pa- cientes que murieron después de 12 meses S. epidermidis 3.3\%, Escherichia coli (E.coli) $3.3 \%$ y $S$. aureus 3.3\% (Tabla 8).

\section{Tabla 8}

Microorganismos encontrados en hemocultivo y cultivo de secreciones de catéter

\begin{tabular}{|crr|}
\hline & Muerte $<\mathbf{1 2}$ meses (\%) & Muerte $\mathbf{1 1 2}$ meses (\%) \\
\hline Hemocultivo & S. aureus 10 & S. aureus 3.3 \\
\hline & S. epidermidis 3.3 & K. pneumoniae 3.3 \\
\hline Cándida albicans 3.3 & S. epidermidis 3.3 \\
\hline Cultivo de secreción de catéter & S. epidermidis 10 & E. coli 3.3 \\
\hline & S. epidermidis 6.7 & S. aureus 3.3 \\
\hline
\end{tabular}

\section{Complicaciones en pacientes con causa de muerte: sepsis + infección de catéter/sepsis + neumonía}

La infección de catéter como complicación en los pacientes que murieron por esta causa, se presentó en el $71.4 \%$ de pacientes que murieron antes de 12 meses y en el
$66.7 \%$ de los que murieron después de 12 meses; siendo la más frecuente.

Otras complicaciones como la hipotensión intrahemodiálisis, se presentó sólo $28.6 \%$ en los que murieron antes de 12 meses y la HDA solo se presentó en los que murieron después de los 12 meses, en 33.3\% (Tabla 9).

\section{Tabla 9}

Complicaciones de pacientes con causa de muerte sepsis + infección de catéter/sepsis + neumonía

\begin{tabular}{|lrr|}
\hline Complicación & Muerte $<\mathbf{1 2}$ meses & Muerte $\geq \mathbf{1 2}$ meses \\
\hline Infección de catéter & $71.4 \%$ & $66.7 \%$ \\
\hline Hipotensión intrahemodiálisis & $28.6 \%$ & $0 \%$ \\
\hline HDA & $0 \%$ & $33.3 \%$ \\
\hline
\end{tabular}

\section{Infarto agudo de miocardio}

Ésta fue la segunda causa de muerte entre los pacientes, $26.7 \%$ en los pacientes que murieron antes de 12 meses y $3.3 \%$ en los que murieron después de 12 meses. La edad promedio de muerte antes de 12 meses fue de 82.9 años y después de 12 meses fue de 86 años.

\section{Riesgo cardiovascular para IAM}

La enfermedad coronaria se presentó sólo en los pacientes que murieron antes de 12 meses con $23.3 \%$. EVC leve en $3.3 \%$ de los pacientes que murieron antes de 12 meses. EVC con hemiplejia $20 \%$ en los que murieron antes de 12 meses. La EVP, se presentó en $6.7 \%$ de los que murieron antes de 12 meses (Tabla 10). 


\section{Tabla | 0}

Riesgo cardiovascular para pacientes con causa de muerte IAM

\begin{tabular}{|lrr|}
\hline & Muerte $<\mathbf{1 2}$ meses (\%) & Muerte $\geq \mathbf{1 2}$ meses (\%) \\
\hline Enfermedad coronaria & 23.3 & 0 \\
\hline EVC leve & 3.3 & 6.7 \\
\hline EVC con hemiplejia & 20 & 0 \\
\hline EVP & 6.7 & 0 \\
\hline
\end{tabular}

\section{Comorbilidades en pacientes con causa de muerte: IAM}

Fue más frecuente la presencia de DM2: $16.7 \%$ en los que murieron antes de 12 meses y $3.3 \%$ en los que murieron después de 12 meses. El tabaquismo se presentó en $23.3 \%$ en los que murieron antes de 12 meses. Se realizó un ECOTT donde se obtuvo la FEVI de todos los pacientes, aquellos que obtuvieron una FEVI menor a
55 se clasificó como ICC, $26.7 \%$ de los pacientes con muerte en menos de 12 meses y $3.3 \%$ de los pacientes que murieron después de 12 meses. En cuanto a la clasificación funcional de ICC de la NYHA, el 10\% de los pacientes que murieron antes de 12 meses, tenían una puntuación de 2, lo que indica que tenían una limitación funcional. La HVI se presentó en $20 \%$ de los pacientes que murieron antes de 12 meses (Tabla 11).

\section{Tabla | |}

\section{Comorbilidades de pacientes con causa de muerte IAM}

\begin{tabular}{|lrr|}
\hline & Muerte $<\mathbf{1 2}$ meses (\%) & Muerte $\mathbf{\geq 1 2}$ meses (\%) \\
\hline DM 2/DM2 con complicaciones & 16.7 & 3.3 \\
\hline Tabaquismo & 23.3 & 0 \\
\hline ICC/FEVI (eco TT) & 26.7 & 3.3 \\
\hline ICC/NYHA & 10 & 0 \\
\hline HVI & 20 & 0 \\
\hline
\end{tabular}

\section{Complicaciones en pacientes con causa de muerte: IAM}

Dentro de las complicaciones, tanto la hipertensión intrahemodiálisis, como la hipotensión intrahemodiálisis, fueron las complicaciones más frecuentes en esta causa de muerte, presentándose 33.3\% cada una, en los pacientes que murieron antes de 12 meses. Otras complicaciones presentadas fueron infección de catéter con $11.1 \%$ tanto en los que murieron antes de 12 meses, como los que murieron después de 12 meses. HDA con $11.1 \%$ en los que murieron antes de 12 meses (Tabla 12). 


\section{Tabla | 2}

Complicaciones de pacientes con causa de muerte por IAM

\begin{tabular}{|lrr|}
\hline Complicación & Muerte $<\mathbf{1 2}$ meses & Muerte $\geq \mathbf{1 2}$ meses \\
\hline Hipertensión intrahemodiálisis & $33.3 \%$ & $0 \%$ \\
\hline Hipotensión intrahemodiálisis & $33.3 \%$ & $0 \%$ \\
\hline Infección de catéter & $11.1 \%$ & $11.1 \%$ \\
\hline HDA & $11.1 \%$ & $0 \%$ \\
\hline
\end{tabular}

\section{EVC hemorrágico}

Ésta fue la tercera causa de muerte, 10\% en pacientes que murieron antes de 12 meses y $3.3 \%$ en pacientes que murieron después de 12 meses. La edad promedio de muerte en pacientes que murieron antes de 12 meses fue de 83.7 años y los que murieron después de 12 meses de 87 años.

\section{Riesgo cardiovascular para EVC hemorrágico}

$10 \%$ de los pacientes que murieron antes de los 12 meses habían presentado enfermedad coronaria. EVC leve se presentó en 3.3\% de los que murieron después de 12 meses. EVC con hemiplejia $3.3 \%$ en pacientes que murieron antes de 12 meses. Sólo los pacientes que murieron antes de 12 meses presentaron EVP con 10\% (Tabla 13).

\section{Tabla | 3}

Riesgo cardiovascular para pacientes con causa de muerte EVC hemorrágico.

\begin{tabular}{|lrr|}
\hline & Muerte $<\mathbf{1 2}$ meses (\%) & Muerte $\mathbf{\geq 1 2}$ meses (\%) \\
\hline Enfermedad coronaria & 10 & 0 \\
\hline EVC leve & 0 & 3.3 \\
\hline EVC con hemiplejia & 3.3 & 0 \\
\hline EVP & 10 & 0 \\
\hline
\end{tabular}

\section{Comorbilidades en pacientes con causa de muerte: EVC hemorrágico}

DM2 fue 3 veces más frecuente en los que murieron antes de 12 meses con $10 \%$ y $3.3 \%$ en los que murieron después de 12 meses. $3.3 \%$ en los que murieron antes de 12 meses presentaban tabaquismo. La clasificación de ICC con FEVI obtenida a través de ECOTT fue de $10 \%$ en los que murieron antes de 12 meses y $3.3 \%$ de los que murieron después de 12 meses. Clasificación de ICC con NYHA obteniendo 2 puntos, sólo fue en pacientes que murieron antes de 12 meses con $6.7 \%$. HVI se presentó con la misma frecuencia 3.3\%, tanto en los pacientes que murieron antes 12 meses, como en los murieron después de 12 meses (Tabla 14). 


\section{Tabla | 4}

Comorbilidades para pacientes con causa de muerte EVC hemorrágico

\begin{tabular}{|lrr|}
\hline & Muerte $<\mathbf{1 2}$ meses (\%) & Muerte $\mathbf{\geq 1 2}$ meses (\%) \\
\hline DM 2/DM2 con complicaciones & 10 & 3.3 \\
\hline Tabaquismo & 3.3 & 0 \\
\hline ICC/FEVI (eco TT) & 10 & 3.3 \\
\hline ICC/NYHA & 6.7 & 3.3 \\
\hline HVI & 3.3 & 3.3 \\
\hline
\end{tabular}

\section{Complicaciones en pacientes con causa de muerte: EVC hemorrágico}

Las complicaciones presentadas fueron hipertensión intrahemodiálisis con $25 \%$ en los que murieron antes y después de 12 meses. Hipotensión intrahemodiálisis presente sólo en $25 \%$ de los que murieron antes de 12 meses. HDA presente sólo en $25 \%$ de los que murieron antes de 12 meses (Tabla 15).

\section{Tabla | 5}

Complicaciones para pacientes con causa de muerte EVC hemorrágico.

\begin{tabular}{|lrr|}
\hline & Muerte $<\mathbf{1 2}$ meses & Muerte $\geq \mathbf{1 2}$ meses \\
\hline Hipertensión intrahemodiálisis & $25 \%$ & $25 \%$ \\
\hline Hipotensión intrahemodiálisis & $25 \%$ & $0 \%$ \\
\hline HDA & $25 \%$ & $0 \%$ \\
\hline
\end{tabular}

\section{Discusión}

La media de sobrevida que se ha descrito y demostrado en países desarrollados es variable entre 12 y 29 meses. $^{8}$ La sobrevida del grupo de pacientes estudiados que ingresaron al programa de HD fue de 8.2 \pm 4.2 meses. A pesar de ser un resultado menor a lo propuesto en otros artículos, se incrementó 5 meses más las sobrevida al compararlo con el promedio de sobrevida en pacientes que no reciben terapia dialítica y se manejan en forma conservadora con TMC (supervivencia de 3 meses). ${ }^{7}$

En este grupo de 30 pacientes, 80\% fallecieron en el primer año. Los factores de riesgo encontrados en los resultados, establecen que las complicaciones intrahemodiálisis promueven un aumento de la mortalidad en los primeros 12 meses. La presencia de complicaciones intrahemodiálisis (hipotensión, hipertensión, HDA e infección de catéter) aumentó hasta 4 veces la probabilidad de muerte por cualquier causa en el primer año. La hipotensión intrahemodiálisis como complicación individual, incrementó 6 veces la mortalidad en el primer año.

La principal causa de muerte fueron procesos infecciosos, favorecidos por el uso de catéter de hemodiálisis, encontrando un incremento de riesgo de muerte por infección de 12 veces sobre los pacientes que usaron fístulas en el primer año. ${ }^{7}$ Es importante establecer que la mayoría de los pacientes necesitaron el uso de catéter de hemodiálisis para inicio de hemodiálisis 
por urgencia dialítica, situación que propicia mayor frecuencia de complicaciones de tipo infeccioso y metabólico al catalogarse como pacientes críticos.

La segunda causa de mortalidad fue IAM, 87,5\% de los pacientes que fallecieron por dicha causa, tenían antecedente de enfermedad coronaria conocida, FEVI menor $55 \%$ en el $77.7 \%$ de ellos, lo que incrementa el riesgo de complicaciones cardiovasculares. ${ }^{11}$

Los resultados obtenidos de los antecedentes cardiovasculares demostraron que el: 1) tabaquismo, 2) enfermedad coro- naria (definida como IAM previo, uso de angioplastía o bypass), 3) EVC con hemiplejia, y la 4) HVI por ECOTT aumentaron la probabilidad de muerte por IAM en el primer año, de acuerdo con los resultados obtenidos para OR (ver apéndice 2).

Las complicaciones intrahemodiálisis (hipotensión, hipertensión, HDA, infección de catéter) incrementan la probabilidad de muerte por IAM en el primer año hasta 2.5 veces. ${ }^{11}$ Por lo que la probabilidad de muerte por IAM en HD durante el primer año está determinado por los antecedentes de patología cardiaca.

\section{Conclusiones}

Como conclusiones finales dentro del grupo de pacientes estudiados, las principales causas de muerte reportadas fueron: 1) procesos infecciosos, 2) IAM y 3) EVC hemorrágico. Los principales factores de riesgo asociados para determinar una mayor probabilidad de muerte en el primer año en el grupo de pacientes mayores de 80 años que reciben hemodiálisis son: 1) complicaciones intrahemodiálisis, 2) infección de acceso vascular y 3) comorbilidad cardiovascular.

Además, se obtuvo evidencia de aumento de probabilidad de muerte en el primer año en aquellos pacientes que presentan antecedentes de 1) enfermedad coronaria 2) tabaquismo, 3) EVC con Hemiplejia, 4) HVI por ECOTT y 5) uso de catéter de hemodiálisis.

Aunque se conoce que la información brindada por el estudio es meramente descriptiva, los datos arrojados pudieran servir como base de información para establecer un perfil de riesgo en aquellos pacientes que presentarían mayor probabilidad de muerte en el primer año; sin embargo debe recalcarse que esta información no puede utilizarse como criterios de contraindicación absoluta para ofrecer este tratamiento y sería necesario llevar a cabo estudios diseñados para análisis de asociaciones.

Por lo tanto creemos que el objetivo del estudio de brindar datos descriptivos de personas mayores de 80 años que inician HD, son cumplidos. Aunque no se revisaron marcadores o variables de mejoría de calidad de vida, se obtienen algunos datos que pueden extrapolarse y servir como factores que impidan a los médicos el desaconsejar el uso de hemodiálisis en personas mayores de 80 años. 


\section{Bibliografía}

1. Méndez - Durán, A., Méndez - Bueno, J., Tapia Yáñez, T., Muñoz Montes, A., \& Aguilar - Sánchez, L. Epidemiología de la insuficiencia renal crónica en México. Diálisis y trasplante. 2010; 31:7-11.

2. S. Berns, J. Hemodialysis in the older adult. UpToDate. 2016.

3. Murtagh, F., Marsh, J., Donohoe, P., EKbal, N., Sheerin, N., \& Harris, F. Dialysis or not? A comparative survival study of patients over 75 years with chronic kidney disease stage 5 . Nephrology Dialysis Transplantation. 2007; 22:1955-1962.

4. Ahmed, S., Addicott, C., Qureshi, M., Pendleton, N., Clague, J.-E., \& Horan, M.-A. Opinions of Elderly People on Treatment for End-Stage Renal Disease. Gerontology. 1999; 45:156-159.

5. Somma, C., Trillini, M., Kasa, M., Gentile, G. Managing End-Stage Renal Disease in the Elderly. Aging Health . 2013; 5: 539-552.

6. Instituto Mexicano del Seguro Social. Tratamiento sustitutivo de la función renal. Diálisis y Hemodiálisis en la insuficiencia renal crónica. CENETEC [Internet] 2017. Disponible en: www.cenetec.salud.gob.mx/ descargas/gpc/CatalogoMaestro/IMSS-727-14DialisisyhemodialisisIRC/727GER.pdf

7. U Mailloux, L., \& L Henrich, W. Patient survival and maintenance dialysis. UptoDate. 2017.

8. G. Couchoud, G., R. Beuscart, J.-B., Aldigier, J.-C., \& J. Brunet, P. Development of a risk stratification algorithm to. ). Kidney International. 2015; 88: 11781186.

9. M. Cohen, L., Ruthazer, R., H. Moss, A., \& J. Germain, M. Predicting Six-Month Mortality for Patients Who Are on Maintenance Hemodialysis. Clinical Journal of the American Society of Nephrology. 2010; 5: 72-79.

10. Beddhu, S., J. Bruns, F., Saul, M., \& Seddon, P. Simple Comorbidity Scale Predicts Clinical Outcomes and Costs in Dialysis Patients. The American Journal of Medicine,. 2000; 108: 609-613.

11. S Berns, J. Complications of hemodialysis in the older patient. UptoDate. 2016 